\title{
Spectral Shape Decomposition by Using a Constrained NMF Algorithm
}

\author{
Foteini Fotopoulou and Emmanouil Z. Psarakis \\ Department of Computer Engineering \& Informatics, University of Patras, \\ Rion-Patras, Greece
}

\begin{abstract}
In this paper, the shape decomposition problem is addressed as a solution of an appropriately constrained Nonnegative Matrix Factorization Problem (NMF). Inspired from an idealization of the visibility matrix having a block diagonal form, special requirements while formulating the NMF problem are taken into account. Starting from a contaminated observation matrix, the objective is to reveal its low rank almost block diagonal form. Although the proposed technique is applied to shapes on the MPEG7 database, it can be extended to 3D objects. The preliminary results we have obtained are very promising.
\end{abstract}

\section{Introduction}

Shape decomposition constitutes of a vital procedure in the field of computer vision, that is able to distinguish the different components of the original object and split it into meaningful ones. Meaningful components are defined as parts that can be perceptually distinguished from the remaining object. In this paper the shape decomposition problem is addressed and a novel decomposition technique is proposed, which solves the above mentioned problem as a special case of the well known NMF situation, using spectral analysis as a head-start. From algebraic perspective, the formulation of NMF can be regarded as decomposing the original matrix into two factor matrices, incorporating the nonnegativity requirement. Far beyond this mathematical exploration, the notion underlying the NMF is closely related to the human perception mode, as perception of the whole is achieved by perception of its parts [?]. For an extended review in the NMF we urge the interested reader to look at [?].

Let us consider a plane curve, that describes a shape boundary, to be defined from the path traced by the following $N$ position vectors:

$$
\mathbf{r}(i)=(x(i), y(i)), i=1,2, \cdots, N .
$$

Then, we can construct the following Visibility Graph $\mathcal{G}_{V}=(\mathcal{V}, \mathcal{E}, \mathcal{W})$, where $\mathcal{V}, \mathcal{E}, \mathcal{W}$ are the nodes and edges sets and a binary weighted matrix respectively. More precisely, in this graph model of the plane curve, nodes' set $\mathcal{V}$ is defined as follows:

$$
\mathcal{V}=\{\mathbf{r}(i), i=1,2, \cdots, N\},
$$


and the $w_{i j}$ element of the $N \times N$ matrix $\mathcal{W}$ can be defined as follows:

$$
w_{i j}= \begin{cases}1, & \text { if nodes } i, j \text { are visible } \\ 0, & \text { otherwise }\end{cases}
$$

where nodes $i, j$ are considered as visible if, the following Visibility Rule hold:

$-\mathcal{V R}$ : The connecting edge $\epsilon_{i j}$ is totally located inside the plane curve.

The $\mathcal{G}_{V}$ of the camel (Figure 1(a)), obtained by the application of the above mentioned Visibility Rule, is depicted in Figure 1(b) As it is obvious the structure of this matrix does not facilitate shape partitioning. An ideal matrix for shape decomposition would have the form of an almost block-diagonal similarity matrix [?], where its non-overlapping blocks could represent the shape's parts, in a sequential manner as it is shown in Figure 1(c). The potential gaps between the blocks denote parts of the shape that do not constitute a group. Such parts are for example those between the camel's hunches, legs etc (see Figure 1(d)] . The basic idea behind this idealization is that each shape component can be

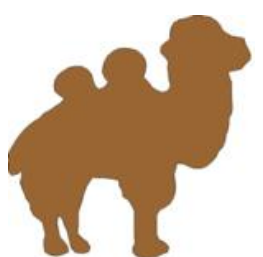

(a)

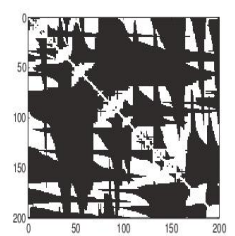

(b)

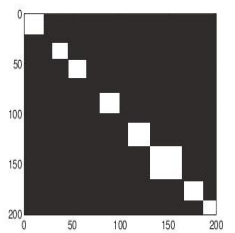

(c)

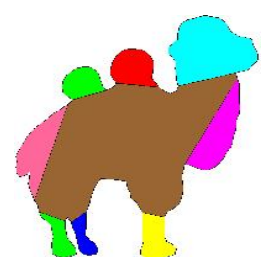

(d)

Fig. 1. The camel-shape (a). The corresponding $\mathcal{G}_{V}$ (b). An ideal block diagonal matrix for the camel shape decomposition (c), and the resulting shape decomposion (d).

represented by a block in the respective block-diagonal matrix. Thus, the shape decomposition problem can be restated as follows: Given the visibility matrix $\mathcal{W}$ construct a block diagonal matrix that best approximates the desired form, which is the objective of our paper.

In an attempt to achieve a rough approximation of the desired block diagonal form, a restriction is imposed to the $\mathcal{W}$ which allows visibility only to a neighborhood of size $n$ on both sides of its main diagonal. In order to calculate a proper radius- $n$ can be adopted any hierarchical method or the method of [?], which we will adopt in this paper.

Constrained $\mathcal{G}_{V}$ resulting from the original $\mathcal{G}_{V}$ of the camel shape depicted in Figure 2(a), are shown in Figure 2(b) 2(d) for three (3) different values of radius $n$. We are expecting that different values of neighborhood radius, result in different decompositions of the candidate shape, which becomes obvious in Figure 3 . It is apparent that none of them is perceptually meaningful, as in none 
of them the "optimal" $n$ value is used. For futher details, please refer to [?]. From now and on we will refer to the redefined $\mathcal{G}_{V}$ as $\hat{X}$.

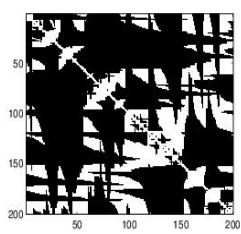

(a)

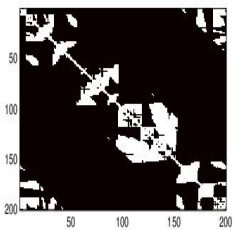

(b)

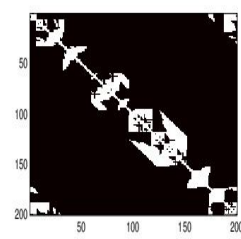

(c)

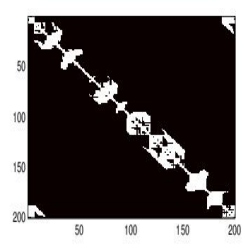

(d)

Fig. 2. Initial $\mathcal{G}_{V}$ (a) and $n$-conditioned $\mathcal{G}_{V}$ for different values of $n$ : 40 (b), 30 (c) and $10(\mathrm{~d})$.

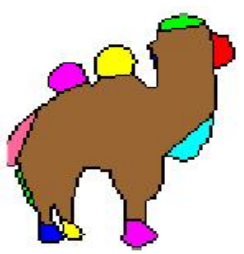

(a)

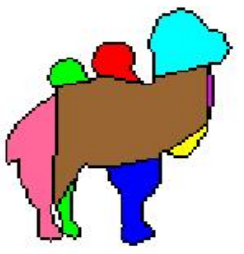

(b)

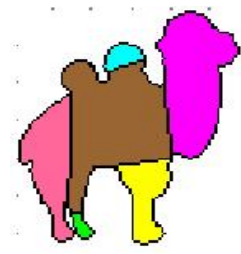

(c)

Fig. 3. Different decompositions of the camel shape corresponding to different values of neighborhood radius $n$ (10, 30 and 40 respectively).

The remainder of the paper is organized as follows: In Section 2 related work is briefly reviewed. In Section 3 the proposed shape decomposition problem is formulated, while in Section 4 the proposed shape decomposition method is developed. In Section 5 the results of the experiments we have conducted in the MPEG7 shape database are presented. In addition a comparison to the matrix completion problem is provided. Finally, the paper concludes at Section 6 .

\section{Related Work}

The task of determining shape's parts is a difficult task due to the involvement of the human perception. However, there exist some generic perception rules examined in psychologist science, with the short cut rule [?], the minima rule [?] and the convexity rule [?,?] to be the most popular among them. Methods for shape decomposition such as [?,?,?,?,?] are based on the above mentioned rules. Besides these three popular perception rules, the authors of [?] propose a new one called part-similarity rule. This rule is based on the observation that similar parts of objects have to be decomposed with the same way, although 
they may look different due to deformations. A method based on differential geometry of smoothed local symmetries taylored for decomposing a shape into its meaningful parts was proposed in [?]. Method [?] suggests an hierarchical segmentation by exploiting the multiscale properties of diffusion distance. In [?] the use of a weak-convexity rule, based on "lines-of-sight" is suggested and the shape decomposition problem is solved by using a spectral clustering algorithm. Finally, the method psoposed in [?] originates from a visibility graph, which captures the local neighborhoods and uses an iterative procedure to transform it into a block diagonal matrix.

As already stated in the Introduction, the shape decomposition problem can be formulated as a special case of the NMF problem. The idea of NMF was initiated by Paatero and Taper [?,?] together with Lee and Seung [?], who demonstrated the NMF potential use in parts based representation. As in this paper we will focus on the constrained NMF situation, and especially in cases where the orthogonality constraint is imposed we proceed with a brief discussion among this category.

The orthogonality principle was first employed in [?] and then in [?] the concept of orthogonal NMF was explicitly expressed. Moreover in [?] the equivalence of the orthogonal NMF to k-means clustering, its formulation as a constrained optimization problem and its solution by using a Lagrange multiplier approach were presented. However the resulting multiplicative update rule, suffers from the zero locking problem. One solution than ensures robust convergence of the algorithm thus solving the above mentioned problem was proposed in [?]. Finally, in [?] an orthogonal NMF algorithm with improved clustering capabilities, based on the original Lee and Seung algorithm and [?], was presented.

Finally, several schemes for the initialization of the factor matrices which affect both the convergence rate as well as the quality of the final solution, have been proposed. In particular, alternatives to the random seed initialization scheme [?] based on k-means [?] and svd [?], have been reported in the literature.

\section{Problem Formulation}

The original symmetric nonnegative matrix factorization problem with orthogonality constraints is already known and can be stated as follows:

Given a symmetric nonnegative data matrix $X \in \Re^{N \times N}$, the goal is to decompose it into a product of two nonnegative matrices, so that $X=V S V^{T}$, where $V$ additionally satisfies the orthogonality constraint and $S$ is added to absorb the different scales of the matrices. More formally, the orthogonal NMF problem can be stated by the following optimization problem:

$$
\min _{V}\left\|X-V S V^{T}\right\|_{F}^{2} \text {, s.to } V \geq 0 \text { and } V^{T} V=I,
$$

where $\|A\|_{F}^{2}$ denotes the squared Frobenius norm of matrix $A$. The aim of this paper is to appropriately reformulate the shape desomposition problem into a constrained NMF problem and solve it. Due to the specific binary form of 
the data matrix $\hat{X}$ the shape decomposition problem is differentiated from the above mentioned NMF problem mainly due to the special form of its low rank component. In particular we would like to decompose the binary $n$-constrained visibility matrix $\hat{X}$ into a low rank component, represented by the binary matrix $X$, and a sparse component $E$, that is: $\hat{X}=X+E$, where matrix's $E$ elements take values from the set $\{1,0,-1\}$.

At a first glance, the above mentioned problem resembles to the well known matrix completion problem [?]. However, there exist some significant differences. At first, our input matrix is binary and the desired output low rank matrix must be strictly binary too. Continuing, it is important to highlight that although a matrix completion algorithm aims at a low rank matrix of general form, we are seeking for a block diagonal one. Indeed, as we are going to see in Section 5 , the matrix completion problem is not well fitted to shape decomposition problem. Consequently, our aim is to recover a special form of $X$, from noisy binary observations contained in the matrix $\hat{X}$. By taking into account all the above mentioned, the shape decomposition problem can be stated as follows:

$$
\min _{V, k}\left\|\hat{X}-V_{k} V_{k}^{T}\right\|_{F}^{2},
$$

subject to the following $N+k$ constraints:

$$
\begin{aligned}
& \mathcal{C}_{1}: \mathbf{c}_{j}^{T} \mathbf{i}_{N+1}=2, j=1,2, \ldots, k \text { with } c_{j i}=\left|v_{j i}-v_{j, i-1}\right|, v_{j, 0}=v_{j, N+1}=0 \\
& \mathcal{C}_{2}: V_{k} \mathbf{i}_{k}=\mathbf{i}_{N}^{\epsilon}
\end{aligned}
$$

where $c_{j i}$ denotes the absolute forward difference of the elements of the zero padded eigenvector $\mathbf{v}_{j}, \mathbf{i}_{M}$ the all one's vector of length $M$ and $\mathbf{i}_{M}^{\epsilon}$ an $\epsilon$-perturbed version of this vector with the value of $\epsilon$ expressing the percentage of its zero elements. This special form of $\mathbf{i}_{M}$ vector allows the decomposition matrix to be almost block diagonal if it is required. This fact is in fully accordance with the ideal matrix description mentioned in the Introduction. Note also that because $v_{j i} \in\{0,1\}$, each one of the constraints $\mathcal{C}_{1}$ imposes the desired form of the eigenvectors, while the constraints $\mathcal{C}_{2}$ express the orthogonality of the columns of the binary matrix $V$. Note finally, that the objective function of the constrained optimization problem does not contain the matrix $S$ anymore.

Although, the above stated problem is NP hard, we are going to overcome this difficulty by starting with the eigenanalysis of matrix $\hat{X}$ and properly imposing in the produced eigenvectors the already stated requirements.

\section{The Proposed Spectral Decomposition Method}

Let us consider that the $\hat{X}$ matrix is given. Then, the proposed method aims at appropriately transforming the original visibility matrix into an almost block diagonal one, which can be easily used for the visually meaningful decomposition of the candidate shape.

The first step of the proposed decomposition method consists of the eigenanalysis of the given matrix $\hat{X}$. Specifically, since this matrix is binary and 
symmetric, from the finite dimensional spectral theorem we know that its eigenvalues are real and that it can be diagonalized by an orthonormal matrix $U$, that is $U^{T} U=I$, as follows:

$$
\hat{X}=U \Lambda U^{T}
$$

where matrix $\Lambda$ contains the real eigenvalues of the matrix.

Let:

$$
\Lambda=\Lambda_{+}+\Lambda_{-}
$$

be two diagonal matrices containg the non negative and negative eigenvalues of matrix $\hat{X}$, respectively. Then, the original matrix can be written as follows:

$$
\hat{X}=\hat{X}_{+}+\hat{X}_{-},
$$

where $\hat{X}_{ \pm}=U_{ \pm} \Lambda_{ \pm} U_{ \pm}^{T}$ and $U_{+}, U_{-}$matrices that contain the eigenvectors which correspond to the non negative and the negative eigenvalues respectively. The number of columns of these matrices are denoted by $N_{+}$and $N_{-}$respectively with their sum to be equal to $N$. Based on the orthogonality of the above defined matrices $\hat{X}_{ \pm}$, the following relation holds:

$$
\|\hat{X}\|_{F}^{2}=\left\|\hat{X}_{+}\right\|_{F}^{2}+\left\|\hat{X}_{-}\right\|_{F}^{2} .
$$

Note that matrix $\hat{X}_{+}$constitutes the optimal non-negative definite symmetric approximation of the original matrix $\hat{X}$. In addition, its Singular Value Decomposition coincides with the following decomposition of the matrix $\hat{X}_{+}$:

$$
\hat{X}_{+}=U_{+} \Lambda_{+} U_{+}^{T} .
$$

Although, the above mentioned matrix is not a non-negative matrix as the desired one, it constitutes a better approximation to our ultimate goal which is a block diagonal matrix. Therefore, we are going to use this matrix in the next step of the proposed method.

In the second step of the proposed algorithm the eigenvectors are sorted in descending order according to the absolute value of their projection onto the vector $\mathbf{i}_{N}$. Specifically, let:

$$
\mathbf{p}=U_{+}^{T} \mathbf{i}_{N}
$$

be the projection of the vector $\mathbf{i}_{N}$ onto the matrix $U_{+}$.

By taking into account the definition of the inner product, the unit norm of each eigenvector and the specific form of vector $\mathbf{i}_{N}$, each element of the above defined vector $\mathbf{p}$ can be expressed as follows:

$$
p_{j}=<\mathbf{u}_{j}, \mathbf{i}_{N}>=\sqrt{N} \cos \left(\theta_{j}\right), j=1,2, \ldots, N_{+},
$$

with $<\mathbf{a}, \mathbf{b}>$ and $\theta_{j}$ denoting the inner product of vectors $\mathbf{a}, \mathbf{b}$ and the existing angle between them respectively.

By defining the following matrices:

$$
\begin{aligned}
S & =\operatorname{diag}\left\{\operatorname{sign}\left(\cos \left(\theta_{j}\right)\right), j=1,2, \ldots, N_{+}\right\} \\
\hat{U}_{+} & =U_{+} S
\end{aligned}
$$


Equ. (11) can be equivalently rewritten as follows:

$$
|\mathbf{p}|=\hat{U}_{+}^{T} \mathbf{i}_{N} .
$$

Note also that by taking into account the fact that the Singular Value Decomposition is only unique up to a reflection of each eigenvector, the decomposition of Equ. 10 can be equivalently rewritten as follows:

$$
\hat{X}_{+}=\hat{U}_{+} \Lambda_{+} \hat{U}_{+}^{T} .
$$

Let us now concentrate ourselves on the vector $|\mathbf{p}|$ defined in Equ. (14). As it is clear, each element of this vector constitutes the projection of the redefined eigenvector $\hat{\mathbf{u}}_{j}$ (or equivanently the $j$-th column of the matrix $\hat{U}_{+}$defined in Equ.(13)) to the all one's vector $\mathbf{i}_{N}$. Thus, it makes sense to assume that the value of each element specifies the contribution of the corresponding eigenvector in the reconstruction of this vector. Consequently, let us sort them in descending order into the vector $\left|\mathbf{p}_{S}\right|$, and rearrange accordingly the columns of matrix $\hat{U}_{+}$ to obtain its desired sorted counterpart $\hat{U}_{S+}$.

In the next step, we are going to replace all these $N_{+}$eigenvectors by their binary equivalents. In order to achieve our goal, for each one of the $N_{+}$eigenvectors, a hard thresholding procedure is applied. To this end, let:

$$
\mathcal{I}_{j}=\left\{\min \left(\hat{\mathbf{u}}_{S j}\right): \frac{\max \left(\hat{\mathbf{u}}_{S j}\right)-\min \left(\hat{\mathbf{u}}_{S j}\right)}{L-1}: \max \left(\hat{\mathbf{u}}_{S j}\right)\right\},
$$

be a sequence of length $L$, resulting from the uniform sampling of the range of the $j$-th column of matrix $\hat{U}_{S+}$, that is the range of the eigenvector $\hat{\mathbf{u}}_{S j}$ and let us define the following sequence (of the same length) of binary vectors:

$$
\mathcal{U}_{j}=\left\{\mathbf{v}_{j i}=\operatorname{sign}\left(\left(\hat{\mathbf{u}}_{S j}-T_{i}\right)>0\right), T_{i} \in \mathcal{I}_{j}\right\} .
$$

Note that the above defined set contains all the binary versions of eigenvector $\hat{\mathbf{u}}_{S j}$ after its hard threshoding by $T_{i}$.

Let us now denote by $\mathcal{U}_{A j}$ the subset of the binary vectors that belong into the set $\mathcal{U}_{j}$ defined in Equ. (17) and strictly satisfy the $\mathcal{C}_{1}$ constraints of the optimization problem (5). It is clear that this subset contains all the admissible binary representations of the eigenvector $\hat{\mathbf{u}}_{S j}$. In order to isolate the most characteristic one, let us compute the $l_{0}$ norm of each admissible vector, i.e.:

$$
l_{i}=|| \mathbf{v}_{j i} \|_{0}, i=1,2, \ldots,\left|\mathcal{U}_{A j}\right|
$$

where $\left|\mathcal{U}_{A j}\right|$ denotes the cardinality of set $\mathcal{U}_{A j}$. Then, find out the most frequent element of the above defined sequence $\mathbf{v}_{j}$ and if its $l_{0}$ norm is greater than a predefined minimum admissible value $m_{\ell_{0}}$, consider it as the most representive binary vector of the specific eigenvector. We must stress at this point that the latter restriction puts a down limit to the size of the smallest permitted shape component. Repeat the above described procedure for each one of the $N_{+}$sorted eigenvectors contained in the matrix $\hat{U}_{S+}$. It is clear that after this we end up 
with a subset of $M$ out of the $N+$ binary eigenvectors which strictly satisfy the constraints $\mathcal{C}_{1}$ and their $l_{0}$ norms are greater than the minimum admissible value $m_{\ell_{0}}$. Finally, sort these binary eigenvectors in desceding order according to their $l_{0}$ norm.

Having defined the binirized form of the $M$ eigenvectors, in the last step of the proposed algorithm we impose all the neccesary constraints onto the binary eigenvectors in order to satisfy the constraint $\mathcal{C}_{2}$ of the constrained optimization problem (5). Specifically, each one of the $M$ selected binary eigenvectors is sequentially examined for the satisfaction of the above mentioned constraint. In particular, for each pair of succesive binary vectors their intersection is identified and if it is not empty is substracted from the eigenvector with the smaller $l_{0}$ norm. Keep the resulting vector if its $l_{0}$ norm is greater than $m_{l_{0}}$. Note that by repeating the above described procedure over all the vectors, we end up with $k \leq M$ mutually exclusive binary vectors which sum up to a $\mathbf{i}_{N}^{\epsilon}$ vector and this concludes the proposed technique.

An outline of the proposed algorithm follows.

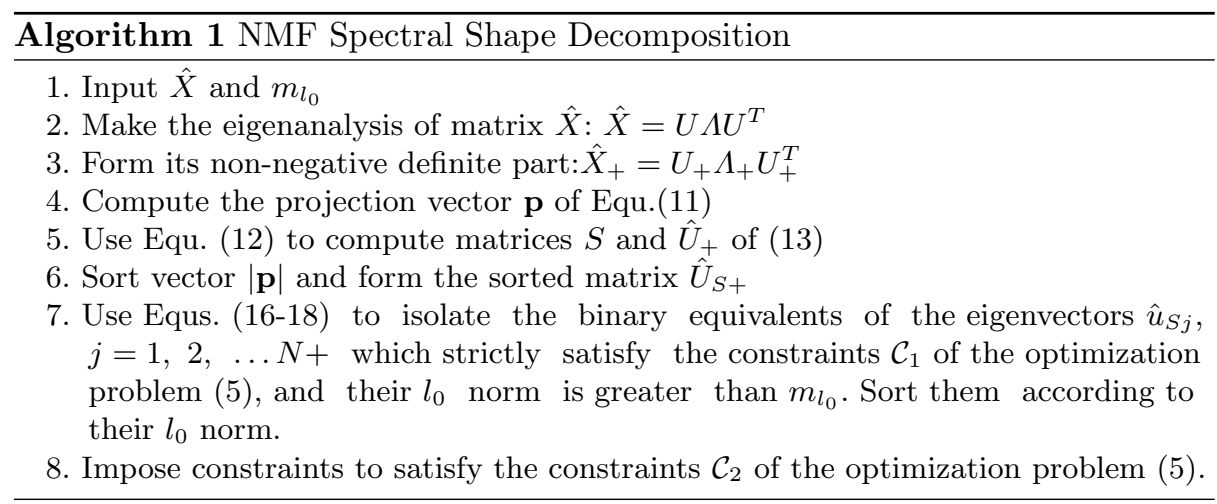

Having completed the presentation of the proposed technique, in the next section we are going to apply it in a number of $2 \mathrm{D}$ shapes and compare its performance against other well-known techniques.

\section{Experimental Results}

\subsection{Shape Decomposition}

In this section we present comparative results obtained by applying the proposed method to several shapes of the MPEG7 shape database part B [?]. All shape contours we used in our experiments were sampled uniformly at 200 points and the size of the smallest permitted shape component, controlled by the value of the In our opinion the propesed method meens $m$, wa be insensitive to the numper and the complexity, of the shape comprengents (see for example the decomposition of the mouse or that of the butterfyy shown in Figure 4). It is evident, that for most shapes the decomposition is meaningfull, while in some situations, parts that obviously could not be separated by a human being, are splitted by the 

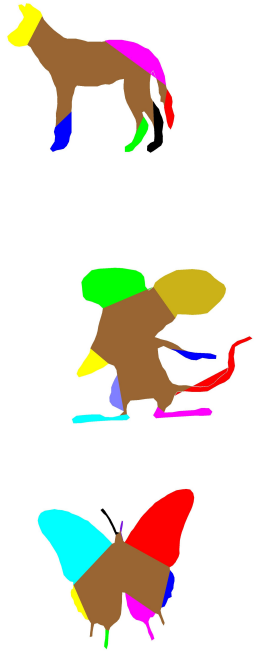
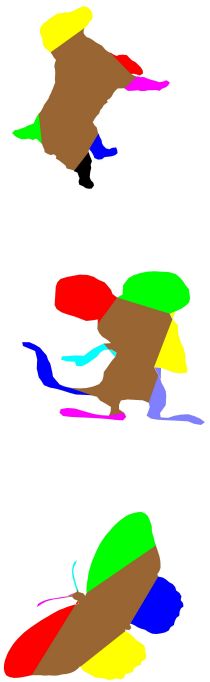
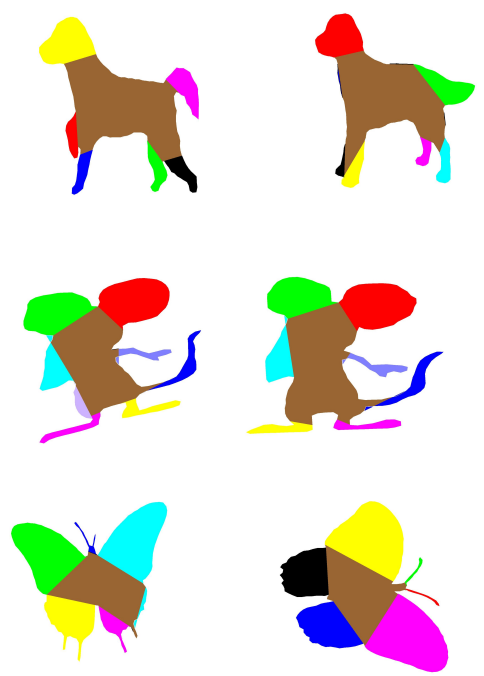

Fig. 4. A sample of decomposed shapes of the MPEG7 database.

algorithm. Specifically, the dog shapes are decomposed into their main parts (i.e. the head, the tail and the legs) in most cases. Moreover, in the mouse the ears, the tails, the hands and the legs are successfully separated. Finally, in the butterfly shapes, the antennas are defined as separate parts, except from the third one. In addition, the wings are well defined, too. An exception is made to the first and third butterfly figures, where the wings are distorted. It is worth mentioning, that the spectral decomposition proposed in this paper, provides satisfactory results achieving at a great degree to recognize most meaningful articulated shape parts, even if they are depicted in different poses, which are acceptable compared to other methods.

To further demonstrate the effectiveness of the proposed decomposition method, we will proceed in showing some comparative results. In particular, we will compare our method to [?],[?], [?] and [?]. The above mentioned results are shown in Figure 5. Since human perception is essential for the evaluation of the produced results, human decompositions are shown in the first column of this figure. For each of the categories (see Figure 5) for which the experiment conducted, humans were asked to decompose the shapes manually into meaningful parts. The results of this experiment were borrowed from [?]. In addition, we should mention that in the fifth column of the Figure 5, where the results of the [?] are depicted, the decomposition does not include the staightening process. As it is apparent, our proposed method succeeds in most cases to successfully approximate the results of the first column. In addition, although in some cases the introduced method identifies some extra components, it succeeds in capturing other ones, that none of the compared methods can do. For example, in the 
elephant shape we have to mention that although its spine is found as a separate shape part, the head is also found as a component, which is in accordance with the perceptual decomposition shown in the first column and in most of the other methods is missed. The same observation holds for the cow's and frog's head. Finally, regarding the beetle shape, our method is the only one among the compared ones that decomposes its legs as separate parts, which confirms the effectiveness of our method to bendable shape parts. Concluding, although the performance of the proposed technique can be characterized as quite good, it could be further improved if a more sophisticated scheme, than that we adopted from [3] is used for structuring the constrained visibility matrix $\hat{X}$. This point is currently under investigation.

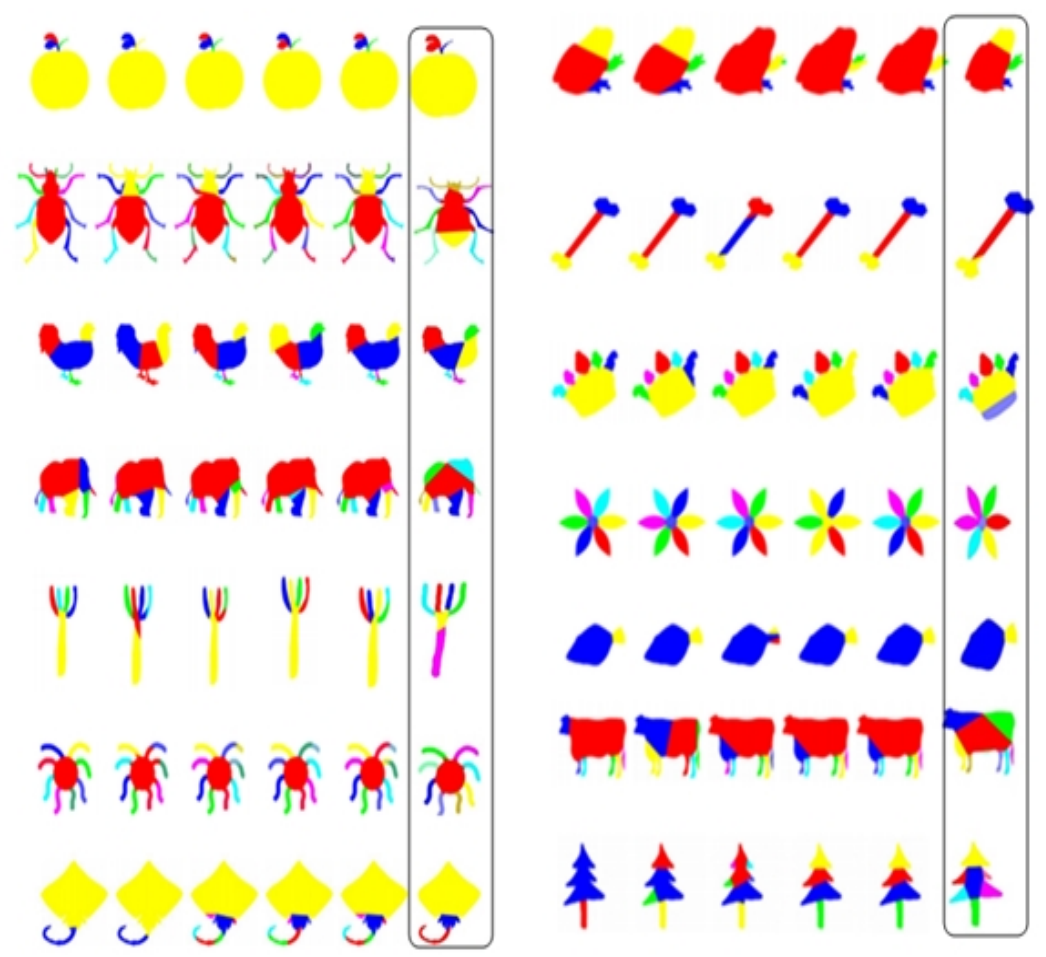

Fig. 5. Examples of the decomposition for 14 categories of the MPEG7 shape database. Human decompositions (1st column) and the results obtained from the application of, [?] (2nd column), [?] (3rd column), [?] (4th column), [?] (5th column), the proposed decomposition technique (last column).

\subsection{Relevance to Other Closely Related Techniques}

Visibility Shape Decomposition: As already mentioned, the idea of the ideal form of an ideal visibility matrix and the use of a constrained visibility matrix as a head-start were borrowed from the VSD method proposed in [3]. Although in [3] the decomposition problem is solved in a totally different way, we consider 
it essential to provide a short comparison between the decomposition results provided by these two methods. Although the experiments showed in the VSD are very promising, there exist many occasions where the resulting decomposition leads to over segmented shapes. This problem is due to the iterative procedure which sometimes fails to capture whole segments as a compact block in the final almost-block diagonal matrix. Therefore, we can observe decompositions where a perceptually expected group is splitted into two smaller ones, with no physical meaning. In Figure (6) two indicative examples of the above stated problem, accompanied by their corresponding block diagonal matrices are shown. As we can observe, the cow's head is over segmented and the same holds for the round part of the apple. On the other side regarding the performance of the proposed method, all the comments we made in the previous subsection are still valid.

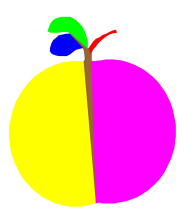

(a)

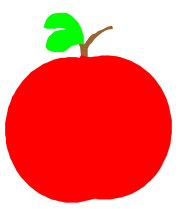

(e)

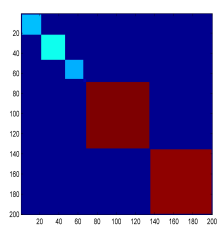

(b)

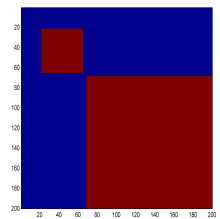

(f)

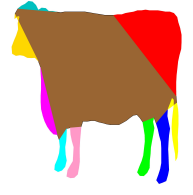

(c)

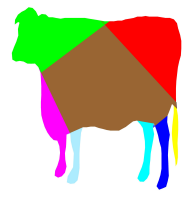

(g)

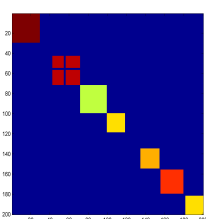

(d)

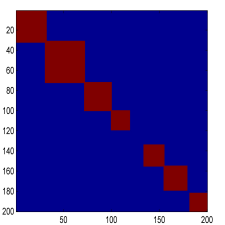

(h)

Fig. 6. Decompositions and block diagonal matrices of the apple and cow shapes resulting from the application of VSD (a-d) and the proposed technique (e-h)

Matrix Completion Problem: Given a corrupted data matrix $D$, the process of matrix completion is to decompose the matrix into a sum of a low-rank matrix $X$ and a sparse matrix $E$. In [?] is shown that, under broad conditions, the optimal solution to the completion problem is given from the solution of the following convex optimization problem:

$$
\min _{X, E}\|X\|_{\star}+\gamma\|E\|_{1} \text {, s.t. } D=X+E
$$

where $\gamma>0$ is a parameter that is used to control the sparseness of the matrix E.

There exist many well-known techniques that can be used for the solution of the constrained optimization problem defined in (12), such as Augmented Lagrange Multiplier (ALM) Method [?], the Accelarated Proximal Gradient (APG) [?], the Dual Method [?], the Singular Value Thresholding [?] to name a few. 
In all the experiments we have conducted we used the IALM and the SVT algorithms ${ }^{1}$ and the results we have obtained confirm that although the Robust PCA [?] methods can be successfully used for solving the matrix completion problem, for the shape decomposition one they seem to be inappropriate.

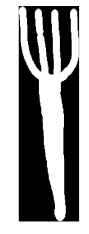

(a)

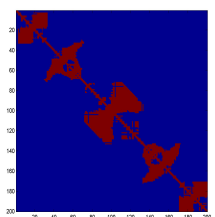

(b)

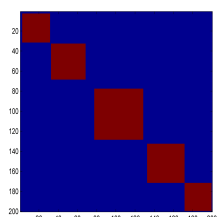

(c)

Fig. 7. The fork shape (a), the rank 193 visibility matrix (b) and the rank 5 block diagonal matrix resulting from the application of the proposed technique (c)

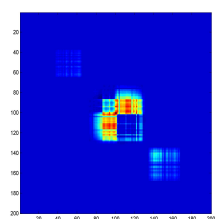

(a)

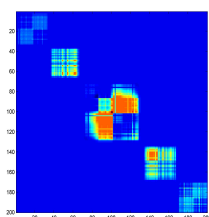

(b)

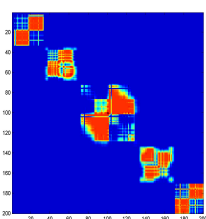

(c)

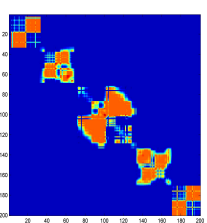

(d)

Fig. 8. IALM decompositions results and the rank of the obtained low rank matrix $X$ for the fork shape for different valious of the parameter $\gamma \cdot \gamma=0.05$, $\operatorname{rank}=5$ (a), $\gamma=0.06, \operatorname{rank}=13$ (b), $\gamma=0.08, \operatorname{rank}=25$ (c), $\gamma=0.1, \operatorname{rank}=46$ (d)

As it is apparent from (19), the greater the value of $\gamma$ is, the sparser the matrix becomes. From the Figure 8 we can observe that as the value of the parameter $\gamma$ increases, the matrix's rank is increased and the resulting matrix escapes the block diagonal form. However, for small values of $\gamma$ some groups seem to reveal, but the desired block diagonal form is not achieved. Specifically, as we can see from Figure 8(a), although the rank of the produced matrix is exactly the same as the rank of the block diagonal matrix resulting from the application of our method (see Figure 7(c)), the IALM algorithm produces a matrix that is not in the desired form. Moreover, by increasing the parameter $\gamma$ we can see in Figures $8(\mathrm{~b}) 8(\mathrm{~d})$ that the constructed matrix seems to somehow approximate

\footnotetext{
${ }^{1}$ In our experiments for the implementation of the matrix completion techniques we have used the matlab codes from http://perception.csl.illinois.edu/ matrix-rank/sample_code.html.
} 
the block diagonal form, however by escaping the low rank constraint. These observations confirm the fact that the completion algorithms in their original form seem to be unsuitable for the shape decomposition problem, as they result in a low rank matrix which has not necessarily the special desired form. The same remarks hold for the SVT completion algorithm.

\section{Conslusions}

In this work, a novel perspective on the shape decomposition issue was proposed. Originating from an eigenanalysis of a constrained visibility matrix, the shape decomposition problem was formulated as a constrained orthogonal NMF one. From the results we obtained it seems that the introduced technique results in perceptually most meaningful decomposition than most of the existing methods. Although the proposed method is applied to a large number of $2 \mathrm{D}$ shapes, extension in $3 \mathrm{D}$ shapes is currently under investigation.

\section{References}

1. Ullman, S.: High-level vision: Object recognition and visual cognition. MIT press (2000)

2. Wang, Y.X., Zhang, Y.J.: Nonnegative matrix factorization: A comprehensive review. Knowledge and Data Engineering, IEEE Transactions on 25 (2013) 13361353

3. Fotopoulou, F., Psarakis, E.Z.: A visibility graph based shape decomposition technique. In: VISAPP (1). (2014) 515-522

4. Singh, M., Seyranian, G.D., Hoffman, D.D.: Parsing silhouettes: The short-cut rule. Perception \& Psychophysics 61 (1999) 636-660

5. Hoffman, D.D., Richards, W.A.: Parts of recognition. Cognition 18 (1984) 65-96

6. : Convexity rule for shape decomposition based on discrete contour evolution. Computer Vision and Image Understanding (73) 441

7. Walker, L.L., Malik, J.: Can convexity explain how humans segment objects into parts? Journal of Vision 3 (2003) 503-503

8. Lien, J.M., Amato, N.M.: Approximate convex decomposition of polygons. In: Proceedings of the twentieth annual symposium on Computational geometry, ACM (2004) 17-26

9. Liu, H., Liu, W., Latecki, L.J.: Convex shape decomposition. In: Computer Vision and Pattern Recognition (CVPR), 2010 IEEE Conference on, IEEE (2010) 97-104

10. Ren, Z., Yuan, J., Li, C., Liu, W.: Minimum near-convex decomposition for robust shape representation. In: Computer Vision (ICCV), 2011 IEEE International Conference on, IEEE (2011) 303-310

11. Jiang, T., Dong, Z., Ma, C., Wang, Y.: Toward perception-based shape decomposition. In: Computer Vision-ACCV 2012. Springer (2013) 188-201

12. Ma, C., Dong, Z., Jiang, T., Wang, Y., Gao, W.: A method of perceptual-based shape decomposition. In: IEEE International Conference on Computer Vision, ICCV 2013, Sydney, Australia, December 1-8, 2013. (2013) 873-880

13. Mi, X., DeCarlo, D.: Separating parts from 2d shapes using relatability. In: Computer Vision, 2007. ICCV 2007. IEEE 11th International Conference on, IEEE (2007) 1-8 
14. De Goes, F., Goldenstein, S., Velho, L.: A hierarchical segmentation of articulated bodies. In: Computer graphics forum. Volume 27., Wiley Online Library (2008) $1349-1356$

15. Asafi, S., Goren, A., Cohen-Or, D.: Weak convex decomposition by lines-of-sight. In: Computer Graphics Forum. Volume 32., Wiley Online Library (2013) 23-31

16. Paatero, P., Tapper, U.: Positive matrix factorization: A non-negative factor model with optimal utilization of error estimates of data values. Environmetrics 5 (1994) $111-126$

17. Paatero, P.: Least squares formulation of robust non-negative factor analysis. Chemometrics and intelligent laboratory systems 37 (1997) 23-35

18. Lee, D.D., Seung, H.S.: Algorithms for non-negative matrix factorization. In: Advances in neural information processing systems. (2000) 556-562

19. Li, S.Z., Hou, X., Zhang, H., Cheng, Q.: Learning spatially localized, parts-based representation. In: Computer Vision and Pattern Recognition, 2001. CVPR 2001. Proceedings of the 2001 IEEE Computer Society Conference on. Volume 1., IEEE (2001) I-207

20. Ding, C., Li, T., Peng, W., Park, H.: Orthogonal nonnegative matrix trifactorizations for clustering. In: Proceedings of the 12th ACM SIGKDD international conference on Knowledge discovery and data mining, ACM (2006) 126-135

21. Ding, C.H., He, X., Simon, H.D.: On the equivalence of nonnegative matrix factorization and spectral clustering. In: SDM. Volume 5., SIAM (2005) 606-610

22. Lin, C.J.: On the convergence of multiplicative update algorithms for nonnegative matrix factorization. Neural Networks, IEEE Transactions on 18 (2007) 1589-1596

23. Mirzal, A.: A convergent algorithm for orthogonal nonnegative matrix factorization. Journal of Computational and Applied Mathematics 260 (2014) 149-166

24. Boutsidis, C., Gallopoulos, E.: Svd based initialization: A head start for nonnegative matrix factorization. Pattern Recognition 41 (2008) 1350-1362

25. Candès, E.J., Li, X., Ma, Y., Wright, J.: Robust principal component analysis? Journal of the ACM (JACM) 58 (2011) 11

26. Latecki, L.J., Lakamper, R., Eckhardt, T.: Shape descriptors for non-rigid shapes with a single closed contour. In: Computer Vision and Pattern Recognition, 2000. Proceedings. IEEE Conference on. Volume 1., IEEE (2000) 424-429

27. Gopalan, R., Turaga, P., Chellappa, R.: Articulation-invariant representation of non-planar shapes. In: Computer Vision-ECCV 2010. Springer (2010) 286-299

28. Lin, Z., Chen, M., Ma, Y.: The augmented lagrange multiplier method for exact recovery of corrupted low-rank matrices. arXiv preprint arXiv:1009.5055 (2010)

29. Lin, Z., Ganesh, A., Wright, J., Wu, L., Chen, M., Ma, Y.: Fast convex optimization algorithms for exact recovery of a corrupted low-rank matrix. Computational Advances in Multi-Sensor Adaptive Processing (CAMSAP) 61 (2009)

30. Cai, J.F., Candès, E.J., Shen, Z.: A singular value thresholding algorithm for matrix completion. SIAM Journal on Optimization 20 (2010) 1956-1982

31. Wright, J., Ganesh, A., Rao, S., Peng, Y., Ma, Y.: Robust principal component analysis: Exact recovery of corrupted low-rank matrices by convex optimization. In: Proc. of Neural Information Processing Systems. Volume 3. (2009) 